\title{
Propionibacterium acnes endocarditis on an annuloplasty ring in an adolescent boy
}

\author{
W Y Vanagt, W J Daenen, T Delhaas
}

Heart 2004;90:e56 (http://www.heartjnl.com/cgi/content/full/90/9/e56). doi: 10.1136/hrt.2004.039180

Propionibacterium acnes, a constituent of the human cutaneous flora, infected both the native mitral valve and a Carpentier mitral annuloplasty ring in an adolescent patient. In the case of culture negative endocarditis, the incubation period of blood cultures should be prolonged to identify this pathogen.

$\mathrm{P}$ ropionibacterium acnes is well known as a common constituent of the skin flora. This Gram positive anaerobe is generally considered to be non-invasive, and its presence in blood cultures is often classified as "skin contamination". However, $P$ acnes can cause endocarditis on prosthetic as well as on native valves. We report on an adolescent patient with $P$ acnes endocarditis eight months after valvoplasty and placement of a mitral Carpentier ring for mitral valve prolapse.

\section{CASE PRESENTATION}

An adolescent boy with severe acne conglobata and a history of scoliosis, umbilical herniation repair, and adenotomy was referred to our paediatric cardiology outpatient clinic at the age of 10 years for evaluation of a systolic murmur. Cardiac evaluation showed a mild mitral valve prolapse and grade III mitral valve regurgitation. Over the following years, he remained free of symptoms but developed significant left atrial and ventricular dilatation caused by mitral valve prolapse, predominantly of the posterior leaflet. Still symptom-free, he underwent cardiac surgery at the age of 15 years because his left ventricular end diastolic diameter had increased from 55 to $70 \mathrm{~mm}$. During the surgical procedure, a quadrangular resection of the prolapsing posterior leaflet and a mitral valvoplasty were performed, with placement of a $32 \mathrm{~mm}$ Carpentier mitral ring (Edwards Lifesciences, Irvine, California, USA). There were no complications, neither during the surgical procedure nor at the time of postoperative recovery.

Eight months after this initial procedure, the patient presented with complaints of increasing fatigue and decreasing exercise tolerance. At physical examination, he was afebrile and in good general physical condition. His weight and length were normal for his age. Auscultation showed only a grade II/VI mitral regurgitation murmur without diastolic murmurs and no precordial thrill was palpable. The examination of the lungs, abdomen, and extremities was completely normal. On the skin, widespread acne lesions were noted, without any signs of Osler nodes, Janeway lesions, splinter haemorrhages, or erythema marginatum.

The ECG showed a sinus rhythm of 70 beats/min, left ventricular hypertrophy, and normal repolarisation.

Echocardiography showed massive central mitral regurgitation. The anterior leaflet moved normally but the movement of the posterior leaflet was limited. There were no signs of either thrombus formation or endocarditis and the artificial ring (Carpentier $32 \mathrm{~mm}$ ) seemed intact. The left atrial (left atrial to aorta ratio $54: 34 \mathrm{~mm}$ ) and left ventricular diameters (end diastolic $78 \mathrm{~mm}$, end systolic $54 \mathrm{~mm}$ ) were enlarged as compared with previous data.

$\mathrm{C}$ reactive protein was $<3 \mathrm{mg} / \mathrm{l}$ and a full blood count with leucocyte differentiation showed no abnormalities. Multiple haemocultures (both aerobe and anaerobe) were taken and remained sterile for five days. Because clinical, echocardiographic, and laboratory data did not indicate the presence of endocarditis, no request was made to the bacteriologist to prolong the incubation period of the blood cultures.

The patient was referred for cardiac surgery because of his symptomatic massive mitral regurgitation. Intraoperatively, detachment of the artificial ring was observed at the level of the septal leaflet. Furthermore, the anterior leaflet was dysplastic and the chordae tendineae had ruptured. Because of the suspicion of endocarditis and the dysplastic character of the valve, the entire valve and annuloplasty ring were removed and an artificial valve (Carbomedics $33 \mathrm{~mm}$, Carbomedics Inc, Austin, Texas, USA) was inserted. Despite multiple negative haemocultures, cultures of both the excised mitral valve and the Carpentier ring were positive for $P$ acnes. The patient was successfully treated with intravenous amoxicillin (150 mg/kg/day) for six weeks and postoperative recovery was uncomplicated. On echocardiography, the artificial valve functioned normally, with no regurgitation or perivalvar leak.

After completion of the intravenous antibiotic treatment, further treatment with oral minocycline $100 \mathrm{mg}$ /day was started.

\section{DISCUSSION}

Information on the occurrence of endocarditis caused by $P$ acnes in the literature is sparse, especially in the field of paediatric and adolescent cardiology. The clinical presentation of patients varies widely: the majority are febrile ${ }^{12}$ and have no peripheral stigmata of endocarditis. The patient in this report remained afebrile until the time of surgery, and his symptoms were milder than those described in the majority of other case reports.

$P$ acnes has been reported to affect artificial valves ${ }^{1-3}$ and pacemaker leads ${ }^{4}$ and can be accompanied by abscess formation, dehiscence of the artificial valve, and cardiac failure in severe cases. ${ }^{3}{ }^{5}$ Occasionally, a normal native valve can also be infected, ${ }^{5}$ especially in immunocompromised hosts. ${ }^{6}$ To the best of our knowledge, there are no previous reports of $P$ acnes endocarditis on an annuloplasty ring.

$P$ acnes is a slow growing germ, which is a possible explanation for the false negative results on repeated blood cultures in this case, where $P$ acnes was identified only on the excised mitral valve and artificial ring. Several authors have advocated prolonged incubation of blood cultures ( $>5$ days) to identify this pathogen in the case of culture negative endocarditis. $^{235}$ The difficult isolation as well as the 
tendency to discard a single positive culture as a skin contaminant may contribute to underestimation of the rate of occurrence of $P$ acnes endocarditis. ${ }^{1}$

In the adolescent population, acne lesions are very common. At the time of cardiac surgery, we advocate a more aggressive use of prophylactic and postoperative antibiotics for patients with acne lesions and valvar abnormalities. Furthermore, $P$ acnes should be regarded as a causative pathogen in patients with intracardiac foreign materials and culture negative endocarditis.

\section{Authors' affiliations}

W Y Vanagt, T Delhaas, Department of Paediatrics, Division of Paediatric Cardiology, University Hospital Maastricht, Maastricht, the Netherlands

W J Daenen, Department of Cardiothoracic Surgery, University Hospital Leuven, Leuven, Belgium
Correspondence to: Dr Ward Y Vanagt, Department of Paediatrics, University Hospital Maastricht, PO box 5800, 6202 AZ Maastricht,

Netherlands; ward.vanagt@fys.unimaas.nl

\section{REFERENCES}

1 Chakour M, Revel F, Godreuil C, et al. Infectious endocarditis due to Propionibacterium acnes on a mechanical heart valve and cardiac stimulator electrode. Presse Med 2002;31:1414.

2 Durupt S, Boibieux A, Ballet-Mechain M, et al. Propionibacterium acnes infectious endocarditis. Presse Med 1998;27:1839-41.

3 Huynh $\pi$, Walling $A D$, Miller MA, et al. Propionibacterium acnes endocarditis. Can J Cardiol 1995;11:785-7.

4 Zedrwitz-Liebenstein K, Gabriel H, Graninger W. Pacemaker endocarditis due to Propionibacterium acnes. Infection 2003:31:184-5.

5 Mohsen AH, Price A, Ridgway E, et al. Propionibacterium acnes endocarditis in a native valve complicated by intraventricular abscess: a case report and review. Scand J Infect Dis 2001;33:379-80.

6 Moreira AL, Haslett PA, Symmans WF. Propionibacterium acnes as the cause of endocarditis in a liver transplant recipient. Clin Infect Dis 2000;30:224-6. 